\title{
On examples in mathematical thinking and learning
}

\author{
Samuele Antonini • Norma Presmeg • \\ Maria Alessandra Mariotti · Orit Zaslavsky
}

Accepted: 6 April 2011/Published online: 20 April 2011

(C) FIZ Karlsruhe 2011

\begin{abstract}
The importance of examples and exemplification in mathematical thinking, learning and teaching, is well recognized by mathematicians, epistemologists and mathematics educators. In the collection of papers on these topics presented in this issue, we aim to contribute to the debate on this theme, proposing original studies carried out from different approaches and perspectives, and linked to other relevant topics within mathematics education.
\end{abstract}

\section{Introduction}

Mathematicians explicitly suggest the use of examples in problem solving (Polya 1945), and in teaching, providing collections of examples and counterexamples in different

S. Antonini ( $\square)$

Dipartimento di Matematica, Università di Pavia, Pavia, Italy

e-mail: samuele.antonini@unipv.it

N. Presmeg

Mathematics Department, Illinois State University,

Normal, USA

e-mail: npresmeg@msn.com

M. A. Mariotti

Dipartimento di Scienze Matematiche ed Informatiche,

Università di Siena, Siena, Italy

e-mail: mariotti21@unisi.it

O. Zaslavsky

Department of Teaching and Learning, Steinhardt School

of Culture, Education, and Human Development,

New York University, New York, USA

O. Zaslavsky

Department of Education in Technology and Science,

Technion, Haifa, Israel

e-mail: oritrath@gmail.comz area of mathematics (see, for instance, Gelbaum and Olmsted 1964). In the last decade, relevant studies in mathematics education have focussed on examples, many papers have been published, and some working groups and conferences have been devoted to this topic. The Research Forum Exemplification: the use of examples in teaching and learning mathematics at the 30th Annual Meeting of the International Group for the Psychology of Mathematics Education (PME 30) (Bills et al. 2006) in Prague, and the subsequent Special Issue of Educational Studies in Mathematics (Bills and Watson 2008) dedicated to examples, have established 'exemplification' as an area of research.

Today, the research investigations on examples are living an intense and productive period. An international workshop hosted by University of Siena on The Role of Mathematical Examples in Argumentation \& Proof, and in Conceptualization \& Definition was organized in 2007 by Antonini, Zaslavsky and Mariotti, with the goal of promoting collaborations among researchers interested in exemplification, conceptualization, argumentation and proof.

The papers collected in this issue sprout from the contributions proposed in that meeting and developed in following through the discussions among the participants.

\section{Examples construction processes}

Giving examples of mathematical objects can be a complex task for students as well as for teachers (Zaslavsky and Peled 1996), but in spite of its complexity such a task is rich of potentialities from an educational point of view. In particular, asking students to construct their own examples is recognized as a powerful tool in mathematics education (Watson and Mason 2005). As suggested by Dahlberg and Housman, "it may be beneficial to introduce students to 
new concepts by requiring them to generate their own examples or have them verify and work with instances of a concept before providing them with examples and commentary" (Dahlberg and Housman 1997, pp. 297-298).

The relevance of learners' generating examples and the importance in mathematics education of the analysis of their processes are summarized in one of the research questions proposed by Bills et al. (2006, p. 125): "what is entailed and revealed by the process of constructing examples and how does construction of examples promote mathematical understanding?" Recently, some researchers highlighted the educational potentialities of the processes of example generation, as a research tool which "provides a 'window' into a learner's mind" (Zazkis and Leikin 2007 , p. 15) that can reveal and promote significant aspects of conceptualization.

The studies reported in the first three articles in this issue address this problematic aspect of exemplification, by analysing example generation processes through different tools and methods and outlining their specificities and educational potentialities.

Taking a phenomenological approach, Mason describes five instances of his own experience of mathematical example construction and tinkering. Mason's description focuses on the solvers' awareness of features of examples that can change (dimensions of possible variation) and of the range where they can vary (range of permissible change). Moreover, the author discusses some pedagogical aspects of example construction in the framework of theories of learning.

The other two articles of this group focus on processes involved in solving tasks in which students are asked to produce an example with certain constraints.

Antonini's analysis is carried out through different tools that emphasize the richness and the complexity of processes. Such a complexity is discussed describing the relations among the semiotic activity, i.e. the production and transformation of signs representing mathematical objects, generation of inferences, metacognitive controls, concept image and concept definition, argumentation and proof. Results highlight the importance of semiotic competences and the control of semiotic activity for producing and treating examples.

An important element affecting example production is students' beliefs about the existence or non-existence of an example. Focusing on this aspect, Furinghetti, Morselli and Antonini set up a model that attempts to grasp the nature of the different stages in the example construction when the solver does not know if the example exists. The model focuses on the dialectic between construction of mathematical objects, generation of a conjecture about the existence or non-existence of a required example and production of argumentation supporting the conjecture.

\section{Examples and conceptualization}

Examples play a pervasive role in concept formation (Dahlberg and Housman 1997), in generalization and abstraction, having different and complex functions in the dynamics between general and particular (Mason and Pimm 1984), and between concept image and concept definition (Tall and Vinner 1981):

Examples can therefore usefully be seen as cultural mediating tools between learners and mathematical concepts, theorems, and techniques. They are a major means for 'making contact' with abstract ideas and a major means of mathematical communication, whether 'with oneself', or with others. Examples can also provide context, while the variation in examples can help learners distinguish essential from incidental features and, if well selected, the range over which that variation is permitted. (Goldenberg and Mason 2008, p. 184)

Two articles of this issue give a direct contribution to the studies about examples in conceptualization, underlining some specificities of exemplification in mathematical thinking. These aspects are investigated in different ways and by different perspectives: while Bardelle and Ferrari focus mainly on the role of language, Vinner deals with processes of concept formation, conjecture formation and verification.

In particular, Bardelle and Ferrari analyse the interplay between definitions and the use of examples in the particular case of the definition of the monotonicity of functions. In an introductory mathematics course for undergraduate students, they asked students to explain the reasons why some examples do or do not satisfy a given definition. The authors discuss the role of the 'pivotal-bridging examples' (Zazkis and Chernoff 2008) in overcoming conflicts concerning students' concept image and they stress the importance of linguistic and semiotic competences in recognizing an example as an instance of a concept.

From a different perspective, Vinner discusses the differences in the use of examples in everyday thought processes and in learning mathematics, and proposes a reflection on aspects of mathematical thinking that are counterintuitive and not spontaneous, showing how many difficulties occur in mathematics because mathematical thinking works in a different way than everyday thinking.

\section{Examples in argumentation and proof}

Examples, argumentation and proof are deeply related. The production and the analysis of examples and counterexamples have been considered central in the dialectic 
between proof and refutation and then in the development of mathematical theories (Lakatos 1976). From a cognitive perspective, mathematics educators have been investigating examples' usage and processes of proving through many approaches. It is well known that students tend to accept a statement as valid on empirical evidence, i.e., if it is true for a certain number of examples (Fischbein 1982). On the other hand, reliance on examples may be helpful in other situations. Examples can play a significant role in generating conjectures (Boero et al. 1999), argumentation (Antonini 2003; Alcock 2004), proof and refutation (Balacheff 1987; Peled and Zaslavsky 1997; Harel and Sowder 1998).

Two articles of this issue investigate the roles of examples in proving processes from different points of view.

In the framework of 'cognitive unity' and in particular with reference to the notion of 'structural continuity' (Pedemonte 2007), Pedemonte and Buchbinder analyse students' generation of argumentation and proof when the conjecture is generated by examining examples. In particular, they investigate and emphasize the role of 'generic examples' (Balacheff 1987) as a bridge between argumentation and proof that realize 'cognitive unity' and, in particular, 'structural continuity'.

With the different goal of identifying activities that can evoke uncertainty and doubt to promote an intellectual need for a general proof, the role of examples in proving processes is also the focus in the article by Buchbinder and Zaslasvky. The authors designed tasks in which a geometrical example satisfying a statement is proposed and students are asked to deal with the generality of that statement, through the question 'is this a coincidence?'. Their findings indicate that the uncertainty motivates the need for proof, and point to the role of examples and counterexamples in the students' proving (or disproving) processes.

\section{Examples in teaching and learning mathematics}

The use of examples in teaching has been proposed and discussed for a long time (see the pioneer work by Rissland Michener 1978). Many studies in mathematics education provided classifications of examples to be used in teaching, identifying aspects that might be significant from an educational point of view as, for instance, the extent of generality (Mason and Pimm 1984; Peled and Zaslavsky 1997), the dimensions of possible variation and the range of permissible change (Goldenberg and Mason 2008), and the potentiality to create and to resolve a cognitive conflict (Zazkis and Chernoff 2008). Some researchers have recently studied the teachers' choice and treatment of examples in classrooms (Watson and Mason 2005; Rowland 2008; Zodik and Zaslavsky 2008). However, the need for further studies was stressed, specifically with the goal of analysing the role of teachers in facilitating learning through carefully chosen examples and consequently designing activities for teacher education:

The specific choice of examples may facilitate or impede students' learning, thus it presents the teacher with a challenge, entailing many considerations that should be weighed. Yet, numerous mathematics teacher education programmes do not explicitly address this issue and do not systematically prepare prospective teachers to deal with the choice and use of instructional examples in an educated way. (Zodik and Zaslavsky 2008, p. 166)

Three articles of this issue take into account the teacher from different perspectives.

Starting from the consideration that an example can exemplify many different mathematical ideas, Watson and Chick investigate the different relations between examples and classes of mathematical objects that they exemplify. From a pedagogical perspective, they analyse the interaction between what is presented to students and students' perceptions and conceptions of what to do with examples. This distance between teachers' goals and students' actions presents teachers with a complex demand in promoting appropriate students' actions with examples. This role is also analysed by Arzarello, Ascari and Sabena who, taking into account both the semiotic and theoretical-logical aspects, propose a model of the dynamics of examples' production and modification in the classroom. The authors show that, in the process of producing examples, the teacher's intervention can be crucial in the generation and modification of an example and in building up the structure of students' example spaces.

The last article changes the perspective and investigates the role of examples in teacher education. The authors, Peled and Balacheff, use carefully and appropriately designed task examples to elicit, identify, and make a change in teachers' conceptions about processes of modelling and problem solving and about the role of mathematics in solving these problems. The article offers both a theoretical analysis and a didactical tool. The theoretical analysis provides a general schema and underlines the importance of solver's control and beliefs, and the nature of the situation context. The tools include tasks to diagnose teachers' conceptions and to facilitate change from a traditional problem solving to a modelling conception.

In summary, the articles of this issue report some original studies and identify some research questions in the classic streams of the investigation on examples and exemplification, and they also contribute to the research in 
some important areas of mathematics education, as conceptualization, argumentation and proof, and teaching and teacher education. The different approaches and perspectives of the contributions highlight some important aspects of learning and teaching mathematics and we hope that readers find stimulating ideas for further investigation of exemplification and related topics.

\section{References}

Alcock, L. (2004). Uses of examples objects in proving. In M. Johnsen Høines \& A. Berit Fuglestad (Eds.), Proceedings of the 28th Conference of the International Group for the Psychology of Mathematics Education (Vol. 2, pp. 17-24). Bergen, Norway: Bergen University College.

Antonini, S. (2003). Non-examples and proof by contradiction. In N. A. Pateman, B. J. Dougherty, \& J. Zilliox (Eds.), Proceedings of the 2003 Joint Meeting of PME and PMENA (Vol. 2, pp. 49-55). Honolulu, Hawai'i: CRDG College of Education, University of Hawai'i.

Balacheff, N. (1987). Processus de preuve et situations de validation. Educational Studies in Mathematics, 18(2), 147-176.

Bills, L., Mason, J., Watson, A., \& Zaslavsky, O. (2006). Research Forum 02. Exemplification: The use of examples in teaching and learning mathematics. In J. Novotná, H. Moraová, M. Krátká, \& N. Stehlíková (Eds.), Proceedings of the 30th Conference of the International Group for the Psychology of Mathematics Education (Vol. 1, pp. 125-154). Prague: Charles University.

Bills, L., \& Watson, A. (2008). Special issue: The role and uses of examples in mathematics education. Educational Studies in Mathematics, 69, 77-79.

Boero, P., Garuti, R., \& Lemut, E. (1999). About the generation of conditionality of statements and its links with proving. In O. Zaslavski (Ed.), Proceedings of the 23rd Conference of the International Group for the Psychology of Mathematics Education (Vol. 2, pp. 137-144). Haifa, Israel: PME.

Dahlberg, R. P., \& Housman, D. L. (1997). Facilitating learning events through example generation. Educational Studies in Mathematics, 33, 283-299.

Fischbein, E. (1982). Intuition and proof. For the Learning of Mathematics, 3(2), 9-24.
Gelbaum, B. R., \& Olmsted, J. M. H. (1964). Counterexamples in analysis. San Francisco: Holden-Day.

Goldenberg, P., \& Mason, J. (2008). Shedding light on and with example spaces. Educational Studies in Mathematics, 69(2), 183-194.

Harel, G., \& Sowder, L. (1998). Students' proof schemes: Results from exploratory studies. In A. Schoenfeld, J. Kaput, \& E. Dubinsky (Eds.), Research on collegiate mathematics education (Vol. 3, pp. 234-283). Providence: American Mathematical Society.

Lakatos, I. (1976). Proofs and refutations: The logic of mathematical discovery. Cambridge: Cambridge University Press.

Mason, J., \& Pimm, D. (1984). Generic examples: Seeing the general in the particular. Educational Studies in Mathematics, 15, 277-289.

Pedemonte, B. (2007). How can the relationship between argumentation and proof be analysed? Educational Studies in Mathematics, 66, 23-41.

Peled, I., \& Zaslavsky, O. (1997). Counter-examples that (only) prove and counter-examples that (also) explain. FOCUS on Learning Problems in Mathematics, 19(3), 49-61.

Polya, G. (1945). How to solve it. Princeton: Princeton University Press.

Rissland Michener, E. L. (1978). Understanding understanding mathematics. Cognitive Science, 2, 361-383.

Rowland, T. (2008). The purpose, design and use of examples in the teaching of elementary mathematics. Educational Studies in Mathematics, 69, 149-163.

Tall, D. O., \& Vinner, S. (1981). Concept image and concept definition with particular reference to limits and continuity. Educational Studies in Mathematics, 12, 151-169.

Watson, A., \& Mason, J. (2005). Mathematics as a constructive activity: Learners generating examples. Mahwah: Erlbaum.

Zaslavsky, O., \& Peled, I. (1996). Inhibiting factors in generating examples by mathematics teachers and student-teachers: The case of binary operation. Journal for Research in Mathematics Education, 27(1), 67-78.

Zazkis, R., \& Chernoff, E. J. (2008). What make a counterexample exemplary? Educational Studies in Mathematics, 68, 195-208.

Zazkis, R., \& Leikin, R. (2007). Generating examples: From pedagogical tool to a research tool. For the Learning of Mathematics, 27(2), 15-21.

Zodik, I., \& Zaslavsky, O. (2008). Characteristics of teachers' choice of examples in and for the mathematics classroom. Educational Studies in Mathematics, 69(2), 165-182. 